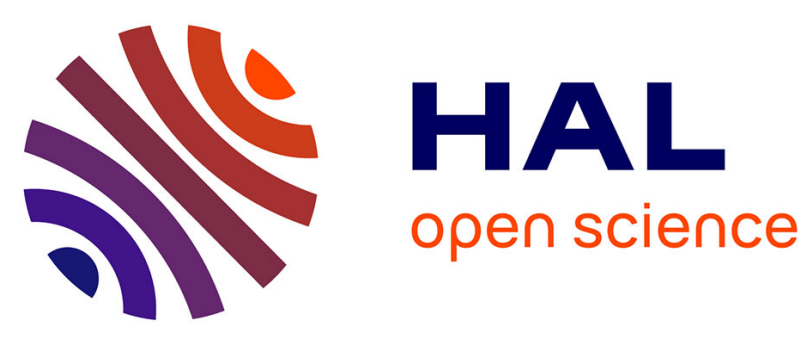

\title{
Manger au Ferlo (Sénégal)
}

Abdou Ka

\section{To cite this version:}

Abdou Ka. Manger au Ferlo (Sénégal): Les charmes risqués des "goûts de la ville". Anthropologie et sociétés, 2020, Habiter le monde: matérialités, art et sensorialités, 44 (1), pp.241-258. 10.7202/1072777ar . hal-03034517

\section{HAL Id: hal-03034517 https://hal.science/hal-03034517}

Submitted on 15 Feb 2022

HAL is a multi-disciplinary open access archive for the deposit and dissemination of scientific research documents, whether they are published or not. The documents may come from teaching and research institutions in France or abroad, or from public or private research centers.
L'archive ouverte pluridisciplinaire HAL, est destinée au dépôt et à la diffusion de documents scientifiques de niveau recherche, publiés ou non, émanant des établissements d'enseignement et de recherche français ou étrangers, des laboratoires publics ou privés. 


\title{
MANGER AU FERLO (SÉNÉGAL)
}

\author{
Les charmes risqués des " goûts de la ville "
}

Abdou Ka

\section{Introduction}

Cet article est axé sur les pratiques alimentaires en milieu rural sénégalais et leurs liens avec la santé des populations. Cette recherche s'inscrit dans un contexte africain où les populations subissent actuellement les répercussions sanitaires et sociales d'une mondialisation touchant à la fois les denrées alimentaires, les idées et les hommes (Macia et al. 2010). Que deviennent les normes locales de comportement alimentaire quand elles sont perturbées par l'accès à des produits manufacturés venus de loin et qu'il faut intégrer (ou pas) au récit alimentaire local ? Dans ce contexte, je me demanderai si une certaine " suralimentation ", au-delà de la délivrance de l'angoisse de faim qu'elle représente, n'est pas source d'une nouvelle forme de dépendance alimentaire.

En milieu rural sénégalais comme ailleurs en Afrique, l'alimentation n'a que peu retenu l'attention des anthropologues et quasiment jamais celle des anthropologues africains. Cette relative absence de travaux scientifiques par ces derniers est intéressante à mettre en évidence en soi. Pendant la colonisation, l'Afrique et les Africains ont été considérés comme des " objets » d'étude privilégiés par les premiers anthropologues (Richards 1932 ; EvansPritchard 1940). Très tôt, toutefois, des auteurs africains tels Jomo Kenyatta (1938) ou Cheikh Anta Diop (1954) ont voulu renverser cette perspective occidentalocentrée.

Dans les années 1940, les premières enquêtes alimentaires en Afrique de l'Ouest ont été lancées au sein de la Mission anthropologique (Pales et Tassin de Saint Peureuse 1955). Elles avaient alors un objectif nutritionnel visant à maximiser la force de travail au sein des colonies. Pour Vincent Bonnecase (2009), si l'on considère d'abord les conditions concrètes dans lesquelles est produit ce savoir, l'une de ses caractéristiques réside dans le peu d'individus sur lequel il repose, qu'il s'agisse des enquêteurs ou des personnes étudiées. $\mathrm{Au}$ moment de sa création, la Mission anthropologique comprend cinq médecins et quatre pharmaciens, tous issus des troupes coloniales françaises. Une enquête consiste à passer huit jours dans une famille, à peser les aliments consommés, à noter leur mode de préparation et à assister au repas. Elle porte donc sur les seuls 
apports visibles. Bonnecase estime que cette recherche occulte les compléments provenant de la chasse ou de la cueillette. Il souligne que certains aliments consommés n'apparaissent ainsi presque jamais, en particulier les fruits.

Aujourd'hui, lorsque les chercheurs se penchent sur l'alimentation en Afrique, ils restent le plus souvent confinés aux questions relatives à l'aide humanitaire ou au développement, renvoyant de ce fait les populations à leur pauvreté économique et à leur précarité (Tercier et Sottas 2000). Depuis plus de 70 ans, les recherches liées à la sécurité et à la transition alimentaires en Afrique et plus particulièrement au Sénégal ne manquent pas. Certains auteurs ont pris l'alimentation ouest-africaine comme objet d'étude, principalement à travers la question des rapports entre alimentation, nutrition et santé. Igor de Garine (1962) a ainsi tracé les balises de ce que " bien manger et bien vivre en Afrique » était censé signifier. Dans « Usages alimentaires dans la région de Khombole (Sénégal) », il estime que si s'alimenter reste une nécessité, elle ne saurait être considérée comme la préoccupation essentielle d'un homme. Il rappelle que le folklore des Sérères comme des Wolofs, ethnies majoritaires à Khombole, abonde en légendes et en dictons qui sanctionnent la gloutonnerie et l'égoïsme alimentaire. Il énumère dans cet article les trois préoccupations qui répondent aux croyances relatives à la valeur nutritionnelle des aliments et à leur utilisation médicale. D'abord, il constate la nécessité de ne pas surcharger un organisme fatigué par un travail de digestion, considéré comme pénible par les mangeurs. Les individus fiévreux et les jeunes mères, les enfants en cours de sevrage ou les jeûneurs reçoivent une alimentation légère destinée à faciliter la digestion. Les aliments épais et gras sont proscrits au profit de bouillies claires comme le ruy (bouillie à base de farine de mil). Ce plat est rapide et facile à préparer, confectionné dès que l'on constate un déséquilibre corporel (maladie, fatigue, etc.). Le ruy est préparé en urgence, pour être consommé immédiatement. Le convalescent reçoit ensuite une alimentation perçue comme énergique, comprenant du lait caillé, du beurre issu de lait de vache, de l'huile de palme, de la viande, du poisson frais.

Dans une perspective plus sociologique, Chelsea Yount (2010) a abordé la question du symbolisme lié à l'alimentation au Sénégal. Pour Yount, le partage du repas familial avec les invités permet aux Dakarois de démontrer leur teranga (hospitalité, en wolof). La teranga est un élément important des règles de politesse couramment manifestées au Sénégal. À travers la teranga, les individus doivent montrer leur générosité à la communauté. Ces caractéristiques personnelles honorent la famille en attestant de la bonne éducation qu'une personne a reçue. D'autres auteurs se sont penchés sur les dynamiques de transformation des habitudes alimentaires en lien avec l'urbanisation (O'Déyé 1985). Pour illustrer ce phénomène, Michèle O'Déyé a pris le cas du chawarma, galette de blé accompagnée d'un mélange de viande grillée et de légumes. Le chawarma, introduit au Sénégal par la communauté libano-syrienne, constituait une nouveauté dans les années 1960 (Crenn et Hassoun 2014). Le chawarma peut 
être considéré comme l'archétype d'une consommation alimentaire alors peu fréquente, car solitaire et hors domicile. De plus, le gros de la clientèle des fastfoods proposant ce sandwich était formé de lycéens, de chauffeurs de taxi, de manœuvres ou de laveurs de voitures. Son prix modique en faisait un prétexte plus qu'une source calorique : excuse pour ne pas rentrer manger chez soi, pour affirmer son indépendance - surtout chez les jeunes — ou, plus simplement, pour se retrouver et discuter.

Dans « Entre brousse, ville et cadre de la globalisation : les paysages alimentaires du Sénégal » (Crenn et al. 2015), nous avons utilisé le concept théorique de "foodscape ", ou " paysage alimentaire », qui nous a permis d'appréhender la construction du monde social sénégalais à travers l'alimentation, tout en redonnant du sens à l'apparent désordre que constitue le domaine de l'alimentation. Ce concept nous a permis de désigner à la fois les dimensions sociale, économique, politique, géographique, symbolique et subjective de l'alimentation, dans une approche dynamique. Les recherches ethnographiques menées dans la ville de Dakar et dans la zone du Ferlo mettent en évidence la manière dont ces deux espaces géographiquement séparés sont sous-tendus par des logiques communes faites de réseaux et de dynamiques socioéconomiques partagés, traversés par des individus (ibid.). Dans la même perspective, j'aborderai en outre dans cet article les représentations sociales relatives à la santé et à l'alimentation tout en mettant l'accent sur des produits spécifiques de l'agro-industrie afin de mettre davantage en lumière les ressentis des pasteurs peuls transhumants du Ferlo.

Le Ferlo, situé dans la zone sahélienne du Sénégal, s'étend sur les régions administratives de Saint-Louis, de Louga et de Matam. Il couvre une superficie de $57269 \mathrm{~km}^{2}$, représentant un peu plus du quart de la superficie totale du pays, soit $29 \%$ du territoire national. Des caractéristiques fondamentales distinguent le Ferlo des autres régions du pays : aridité du climat, prédominance d'une savane arborée et développement de l'élevage extensif. Cet élevage est pratiqué en très grande majorité par les Peuls (Demante 2006). Le Ferlo continue à être présenté par beaucoup de chercheurs et de politiques comme résistant aux changements, vivant sur lui-même dans une sorte d' « autarcie exotique » (Manoli et Ancey 2013). Michel Benoit (1988) qualifie les pasteurs peuls nomades d'adeptes d'une société « libertaire » et « égalitaire». Toujours selon Benoit, en s'éloignant chaque année des vallées avec leurs troupeaux, les Peuls du Ferlo disaient qu'ils allaient vers la brousse vierge, donc vers la liberté d'échapper à la contrainte étatique et la possibilité de vivre leur idéal (ibid.). La mobilité de ces pasteurs peuls a ainsi été analysée essentiellement par beaucoup de chercheurs s'intéressant à ce peuple au travers du nomadisme et de la transhumance, délaissant les migrations urbaines et internationales (Bonfiglioli 1988). Et pourtant, ces migrations jouent un rôle prépondérant dans le processus de changement des pratiques alimentaires. 
Dans cette zone sahélienne très éloignée des grandes villes sénégalaises, l'alimentation n'échappe pas à l'internationalisation croissante des échanges. Sur ce territoire, l'alimentation est aussi marquée comme ailleurs par les rapports sociaux, politiques et économiques, ainsi que par la pensée symbolique. Arjun Appadurai (1996), dans son étude de la mondialisation, analyse la circulation des personnes, des idées et des objets en termes de flux. Ces flux participent d'une manière notable au façonnement des manières de faire et de penser l'alimentation dans le Ferlo. Selon la thèse de la gastro-anomie (Fischler 1979), les normes et les pratiques alimentaires locales perdent de l'importance sous l'influence de la mondialisation. De nouveaux produits, de nouvelles manières de manger, dans des configurations originales, s'associent à des habitudes " anciennes". Dans la même perspective, Jean-Pierre Corbeau (1994) met l'accent sur les différentes formes que peut prendre ce " métissage » alimentaire et insiste sur ce que permet l'anomie : mutations, prise de conscience du patrimoine alimentaire, innovations ${ }^{1}$. Pour Jean-Pierre Poulain et Laurence Tibère (2000), la mondialisation est le résultat de la disparition de certains particularismes, de l'émergence de nouvelles formes alimentaires et de la diffusion à l'échelle transculturelle de certains produits et pratiques alimentaires.

Les conséquences de la grande sécheresse des années 1970, qui avait affecté cinq pays du Sahel - en l'occurrence le Sénégal, le Mali, le Niger, l'ex-Haute-Volta (Burkina Faso) et le Tchad - , vont apporter des changements considérables en ce qui a trait aux pratiques alimentaires. L'agriculture pluviale, la chasse et la cueillette de plantes sauvages alimentaires vont être abandonnées progressivement par les Peuls du Ferlo au profit d'un important élevage de petits ruminants (chèvres et moutons). Ces animaux vont être vendus sur les marchés hebdomadaires afin d'acheter des denrées. De l'autosubsistance, de l'autonomie alimentaire, les populations du Ferlo estiment être passées à la dépendance totale à l'extérieur. Leur survie dépend désormais des marchés hebdomadaires ou louma. Les pasteurs du Ferlo, fortement touchés par la crise climatique, accompagnés de leur bétail, vont découvrir en grand nombre les régions du Sine Saloum et de la Casamance dans leur recherche de pâturages ${ }^{2}$. Ceux qui avaient perdu tout leur bétail se déplacent vers les centres urbains ou les pays limitrophes pour se faire commerçants, ouvriers, garagistes, maçons, couturiers, etc. Leurs rencontres avec d'autres populations et d'autres cultures sont des moments de grandes découvertes sur le plan alimentaire et comportemental (Ka 2016). Dans les villages du Ferlo, les gargotes et les boutiques sont pleines de produits alimentaires importés. L'implantation hebdomadaire des marchés a également considérablement modifié l'offre alimentaire présente dans cette région et, par conséquent, ses pratiques et représentations. Depuis leur arrivée

1. L'anomie peut avoir aussi des facettes négatives. Elle signifie aussi la perte, et non seulement le gain.

2. Selon mes interlocuteurs, ils n'ont pas rencontré de problèmes majeurs avec les populations d'accueil lors de ces grandes transhumances. 
supposée d'Égypte (Lam 1993), les Peuls ne cessent de sillonner le Sahel à la recherche de pâturages et de points d'eau. Cependant, la grande sécheresse des années 1970 a eu une forte influence quant aux changements de leurs pratiques alimentaires. Cette crise climatique a en effet annoncé le début d'une moindre consommation de produits laitiers et d'aliments à base de mil et de viande. Elle est aussi devenue synonyme d'une forte consommation de riz importé et de nombreux autres produits de l'agro-industrie.

L'étude des problèmes de santé liés à la «suralimentation » reste toujours un sujet délicat à aborder en Afrique tant les questions relatives à la faim, à la dénutrition et à la malnutrition dominent les débats (Gado 1993). Pourtant, au Sénégal, l'existence de maladies non transmissibles associées à une alimentation excessive constitue une réalité concrète comme en témoigne l'article de Enguerran Macia, Lamine Gueye et Priscilla Duboz (2016) en démontrant la prévalence importante de l'hypertension artérielle et de l'obésité dans la capitale. Le Ferlo n'échappe pas à cette situation, comme l'a récemment montré une étude menée sur un échantillon représentatif de la population de la commune de Téssékéré, une localité du Ferlo (Duboz et al. 2016). Il est à noter que ces maladies chroniques non transmissibles se développent conjointement avec la disponibilité de produits alimentaires importés par l'intermédiaire des boutiques et des marchés hebdomadaires. Ce phénomène s'inscrit dans un contexte de changement global : la désertification et la raréfaction des pluies ont conduit les populations à abandonner l'agriculture vivrière. Les populations dépendent donc désormais de l'importation, de produits venant de l'extérieur.

Les résultats de cet article seront ici organisés en quatre sous-parties interdépendantes. Tout d'abord, il s'agit dans cette recherche de mettre en lumière, à travers le concept du « triangle du manger » de Jean-Pierre Corbeau (Corbeau et Poulain 2002), le processus des mutations alimentaires afin d'en restituer les transformations ainsi que les permanences dans ce contexte en changement. Par la suite, j'interrogerai les transformations des normes alimentaires dans le Ferlo, notamment en lien avec le développement des marchés hebdomadaires et l'émergence des jardins polyvalents du projet de la Grande muraille verte ${ }^{3}$ dans la région. Enfin, j'aborderai les tensions entre alimentation et santé à travers deux aliments fondamentaux dans la cuisine locale : le riz et les bouillons cubes.

\section{Une « ethnographie du proche »}

En allant faire des enquêtes au Ferlo dans le cadre de mes études de doctorat, je savais qu'une partie de ma famille paternelle y résidait. Une rencontre avec l'un de mes oncles au marché hebdomadaire de Widou Thiengoly ${ }^{4}$

3. La Grande muraille verte est un projet de lutte contre la désertification initié par l'Union africaine. C'est une bande de sept mille kilomètres de long et de quinze kilomètres de large. Elle traverse onze pays africains, de Dakar à Djibouti.

4. Il y était venu pour vendre ses moutons et faire des provisions. 
fut un moment de grandes retrouvailles. C'est lui qui m'a vu en premier ; il m'a abordé et présenté sa femme et ses amis. Il était très heureux de m'avoir retrouvé au cœur du Ferlo sableux. C'était lors de ma première mission dans cette région très éloignée de Dakar (huit heures de route). À partir de ce moment, j'ai pris la décision de faire de l' " ethnographie du proche », en mesurant la difficulté que cela induisait. J'ai déjà une base « experience-near» (proche de l'expérience) par la longue fréquentation de quelques-uns de mes interlocuteurs. La particularité de mon terrain réside ainsi dans mon appartenance à une partie du groupe ethnique étudié. Socialisé par mes parents dans le groupe des Peuls de Dakar depuis mon enfance, $\mathrm{j}$ 'ai grandi par intermittence au contact de certaines des personnes que j'allais interroger. J'ai surtout pesé le pour et le contre : l'accès au terrain était « facile », puisque familier; l'obstacle serait le risque de ne pas être pris au sérieux par des personnes qui m'ont vu grandir. Pour réaliser un tel travail de recherche, il était d'autant plus nécessaire d'interroger mes propres évidences, de remettre en cause ce qui semble aller de soi. Ce travail sur soi me semblait également indispensable pour une enquête par dépaysement, afin de ne pas culturaliser, de ne pas réifier les enquêtés.

Pour cette étude, j'ai utilisé une méthode qualitative, basée sur des entretiens semi-directifs, approfondis, répétés et de longue durée ${ }^{5}$. Ces entretiens semi-directifs ont été complétés par des observations fines et détaillées des différents espaces investis (bourgs, campements ${ }^{6}$, forages d'eau potable, marchés hebdomadaires, gargotes, jardins polyvalents, etc.). Les notes consignées dans mon carnet de terrain lors des observations constituent des données qui m'ont permis d'étoffer et d'affiner les analyses. Le guide d'entretien mis en place pour répondre à ma problématique permettait d'aborder des questions liées aux ressentis, puis aux représentations alimentaires, afin d'éviter toute posture réifiante de la culture alimentaire de cette zone. La méthode dite «boule de neige $^{7} »$ (Combessie 2007) m'a permis de diversifier et d'élargir la population d'enquête afin d'obtenir un échantillon varié. Au total, treize villages ont été observés en profondeur. Des individus résidant dans d'autres villages ont aussi été interviewés au gré des circonstances opportunes : après une réunion dans les bureaux de la Direction des eaux et forêts, chasses et de la conservation des sols $^{8}$, au marché hebdomadaire, après une visite au poste de santé, etc. La grande majorité des personnes interrogées résidaient dans le Ferlo - principalement dans les campements —, mais quelques commerçants colporteurs ont également

5. Les entretiens ont été réalisés dans les langues vernaculaires de cette région (pulaar et wolof) puis retranscrits en français. Tous les entretiens ont été enregistrés à l'aide d'une machine à dicter.

6. Les campements sont les habitations qui constituent les petits villages satellites des bourgs créés à côté des forages.

7. La méthode " boule de neige " consiste à entrer en contact avec de nouveaux enquêtés par l'intermédiaire des personnes interrogées précédemment.

8. La Direction des eaux et forêts, chasses et de la conservation des sols est sous la tutelle du ministère de l'Environnement. Elle est chargée de la protection des ressources naturelles. 
été interviewés. Il est vrai que dans les bourgs, et particulièrement dans les locaux de la Direction des eaux et forêts, des réunions se tiennent fréquemment - qu'il s'agisse de celles organisées par les élus locaux avec les représentants de l'État, les chercheurs que nous sommes, ou de celles tenues avec des représentantes de groupements de promotion féminine gérant les jardins polyvalents du projet de la Grande muraille verte.

\section{Le « triangle du manger » dans le Ferlo sénégalais}

Pour tenter de penser les changements alimentaires dans le Ferlo, je me suis appuyé sur le concept de " triangle du manger » de Corbeau (Corbeau et Poulain 2002). Pour Corbeau, parler de notre alimentation nécessite l'identification d'un mangeur (les populations du Ferlo) consommant un aliment lui-même identifié (le riz, le mil, le pain, les laitages, les légumes frais, etc.) auquel il donne un sens dans une situation particulière (domicile, hors domicile, cérémonie, etc.). Il obtient ainsi les trois sommets d'un « triangle du manger ». Il précise que les mangeurs, de sexes et d'âges différents, de toutes les catégories sociales, de toutes les cultures, s'inscrivent dans ce triangle.

Dans mes recherches, en m'appuyant sur le triangle du manger, $j$ 'insiste sur les mangeurs en tant qu'acteurs de leur destinée alimentaire. Je tente ensuite d'historiciser les pratiques alimentaires contemporaines et les aliments eux-mêmes tout en ayant parfaitement conscience que les données recueillies seront nécessairement à analyser en fonction du contexte d'énonciation et de la situation d'enquête dans laquelle les mangeurs seront observés. J'analyse aussi en quoi les pratiques alimentaires permettent de mettre en évidence les processus d'hybridation culturels et sociaux amorcés dans le Ferlo. Pour Corbeau, parler d'alimentation implique de penser un mangeur identifié achetant, cuisinant, ingérant un aliment ou un plat particulier auquel il donne un sens dans une situation donnée. Nous obtenons ainsi les trois sommets d'un triangle du manger. Selon lui, ces sommets sont en perpétuelle interaction (ibid.). Suivant ce modèle, au Ferlo comme ailleurs, les mangeurs que j'ai interviewés sont donc à la fois producteurs et reproducteurs de normes alimentaires, culinaires et sanitaires selon la disponibilité des produits, la situation vécue et le contexte sociohistorique dans lequel ils s'inscrivent. L'interaction entre des mangeurs et un aliment, à un moment donné, se situe dans le troisième élément du triangle : la situation. Cette dernière va elle-même modifier la manière dont un aliment va être perçu. Par ailleurs, la situation permet aux mangeurs de développer entre eux des formes nouvelles de commensalité avec l'arrivée de nouveaux produits sur les marchés et dans les gargotes. 


\section{Les marchés hebdomadaires et les jardins polyvalents de la Grande muraille verte : vers de nouvelles normes alimentaires et sociales}

\section{La place centrale des marchés hebdomadaires}

Dans les années 1980, l'économie du Sénégal a connu une série d'ajustements structurels. Le désengagement des services de l'État en milieu rural a permis le développement de marchés hebdomadaires gérés par des acteurs locaux (Ninot 2003). J'ai profité des jours de marché dans le Ferlo pour répertorier les différents aliments présents. C'est l'occasion pour les populations de s'approvisionner en denrées alimentaires et autres produits de consommation qu'elles ne trouvent pas toujours dans les commerces des bourgs. Il est à noter également la présence de gargotes tenues par des femmes venues de différents endroits de la région, où elles vendent des sandwichs à base de niébé, de mayonnaise, d'omelette, et des plats de riz au poisson ou à la viande, du mafé, du yassa, etc. (Crenn et Ka 2012.) Les marchés hebdomadaires sont des moments qui permettent de relever le type d'aliments achetés et vendus. Les légumes sont cultivés dans la vallée du fleuve Sénégal et dans les jardins polyvalents de la Grande muraille verte, tandis que le poisson vient de Saint-Louis ou de Thilé Boubacar. Le mil vient du Saloum et du Djolof. Le riz importé vient de Thaïlande ou d'Inde. Le riz local provient des rizières de la vallée du fleuve Sénégal. Les oignons sont importés de Hollande, le thé vert de Chine. On retrouve dans les marchés hebdomadaires des macaronis, du sel, de l'huile végétale raffinée, de l'huile d'arachide traditionnelle (ségal), des bouillons cubes, du vinaigre, du poivre, des bonbons, des biscuits, du sucre en poudre, etc. Les femmes du Ferlo profitent de ces occasions pour vendre des produits laitiers, des fruits sauvages (pain de singe, fruits de Balanites aegyptiaca ${ }^{9}$, jujubes, etc.) et des volailles locales. Les légumes des jardins polyvalents du projet de la Grande muraille verte entrent en concurrence avec ceux de la vallée du fleuve Sénégal.

\section{Des vergers et des potagers au cour du Sahel}

L'Agence nationale de la Grande muraille verte au Sénégal, avec l'appui de ses partenaires tels le Programme alimentaire mondial (PAM) et l'Organisation des Nations Unies pour l'alimentation et l'agriculture (FAO), a aménagé des jardins polyvalents constitués de vergers et de potagers. Elle a installé des systèmes d'irrigation au goutte-à-goutte reliés aux forages d'eau potable, comme le confirment les propos de cette femme : «C'est la Grande muraille verte qui a payé le grillage, les tuyaux du système de goutte-à-goutte. Nous, on n'a rien payé. Même pour le gazole, c'est eux qui payent. » (Femme, 58 ans.) L'accès à ces jardins a permis aux femmes de cultiver des carottes, des aubergines, des pommes de terre, des navets, des melons et des pastèques pendant une bonne partie de la longue saison sèche. D'abord, elles achètent sur place leurs propres

9. Dattier du désert. 
récoltes issues de leurs jardins à bas prix ${ }^{10}$ et le reste est écoulé le jour du marché. Une partie de l'argent issu de la vente des productions est épargnée et déposée à la banque et l'autre sert à acheter du riz qui va être revendu moins cher aux membres du groupement des femmes qui gèrent le jardin polyvalent. Dans les jardins polyvalents et autour se profilent des enjeux sociaux, politiques et économiques très importants pour le Ferlo, notamment l'émergence d'une prise de conscience des femmes responsables de la gestion collective des jardins. Ces périmètres de culture sont des lieux d'intenses interactions entre les femmes des différents campements, de différents groupes ethniques (peuls, wolofs, maures, etc.). C'est là que s'est mise en place une élite féminine influant sur la construction de nouvelles normes alimentaires et sociales sur le territoire du Ferlo. Les jardins polyvalents du projet de la Grande muraille verte constituent un espace où trois types d'acteurs participent aux changements alimentaires : des populations autochtones, des États et des institutions internationales.

\section{Le riz, la céréale de toutes les controverses}

\section{Le riz de l'aide alimentaire, le riz du riche}

Avant la colonisation, les mets à base de riz étaient rares dans la cuisine sénégalaise en général. Cela était dû au faible nombre d'endroits où la consommation alimentaire était traditionnellement basée sur le riz. Jadis ces endroits étaient principalement situés dans la Basse Casamance et, de façon accessoire, dans certaines zones humides du Sénégal oriental et du Saloum. $\mathrm{Au}$ Ferlo, les premiers à avoir accès à cette denrée hautement convoitée furent les chefs de clan (ardo, en pulaar) et les grands propriétaires de bétail (jaarga, en pulaar). Ainsi, ceux que nous avons interrogés racontent qu'il y avait des gens qui passaient la journée chez les ardo ou les jaarga dans le seul but de goûter au riz : " On mangeait du riz depuis très longtemps, mais il n'était pas à la portée de tous. On le mangeait chez ceux qui avaient de gros troupeaux » (femme, 68 ans). D'où provenait ce riz consommé au Ferlo par les populations aisées ? Nos enquêtes révèlent que le riz mangé à cette époque venait essentiellement de l'extérieur. Dans Agriculture et sécurité alimentaire au Sénégal, Boubacar Ba montre que l'Administration coloniale s'efforçait de répondre à la demande alimentaire en important du riz, une importation qui était destinée à approvisionner dans un premier temps la population urbaine, mais aussi la population rurale, accessoirement, surtout quand il y avait des risques avérés de crise alimentaire ( $\mathrm{Ba} 2008$ ).

Pour maintenir sa mainmise sur les grandes personnalités dans cette société pastorale peule clanique et hiérarchisée, le pouvoir colonial leur faisait des dons de riz. Progressivement, cette « arme alimentaire» (Bessis 1981) allait

10. C'est la trésorière ou ses adjointes qui récupèrent l'argent provenant de la vente des récoltes et, après, elles le déposent à la banque. 
devenir un aliment de fête qui prendrait une place importante dans les habitudes alimentaires. Ce qu'il y a de paradoxal ici, c'est que c'est à la fois un produit associé à la richesse (pour les habitants du Ferlo) et à l'aide alimentaire (pour les colons). Tout comme le souligne Sidney Mintz (1991) relativement à la consommation de sucre par la classe ouvrière britannique au XVIII ${ }^{\mathrm{e}}$ siècle, alors que cette substance était autrefois réservée à la noblesse et à la bourgeoisie, la simple consommation du riz permet, dans l'imaginaire, de rejoindre les classes aisées des bourgs ruraux, des grandes villes. Comme l'usage du sucre, celui du riz traduisait à lui seul l'adoption de l'idéologie de la société marchande dans le Ferlo. Cette innovation alimentaire accompagnait en quelque sorte l'histoire du Ferlo, l'histoire de sa relation au monde. La brisure de riz introduite au Sénégal pendant la colonisation a remplacé progressivement le mil à la suite des crises climatiques qui ont fragilisé l'agriculture locale et ouvert la porte aux importations alimentaires. Auparavant, le mil était très présent dans l'alimentation sénégalaise. Chaque terroir avait ses spécialités à base de mil (Crenn et al. 2015).

\section{Une denrée dépréciée par tous, mais consommée par chacun}

Aujourd'hui, au Ferlo, les ménagères préfèrent le riz au mil et particulièrement le riz importé, valorisé pour sa praticité. Le riz importé est plus commode à préparer et symbolise la femme moderne libérée des tâches harassantes qu'exige la préparation des céréales traditionnelles (mil, sorgho). La préparation du mil nécessite beaucoup de travail manuel. Par exemple, pour faire du couscous, les femmes doivent faire plusieurs pilages pour enlever le son et moudre les grains afin d'obtenir la farine de mil. Cependant, beaucoup de mangeurs rencontrés dans le Ferlo jugent que la consommation du riz a un impact négatif sur leur santé. Cette idée est confortée par cet extrait d'entretien : " On ne mange que du riz qu'on ne digère pas. On mange du riz de mauvaise qualité. On le fait parce qu'on n'a pas le choix » (homme, pasteur, 63 ans). Dans le même ordre d'idées, Carla Sarrouy affirme que le riz consommé par les Sénégalais est loin d'être un produit de luxe : la brisure est un sous-produit de la production rizicole, ce qui fait qu'elle est négociée à très bas prix sur la scène internationale (Sarrouy 2010).

Les discours des plus âgés laissent voir que l'abandon de la consommation régulière de mil suscite un sentiment d'amertume. Le souvenir des mets à base de mil consommés au temps du Ferlo fertile leur rappelle un âge d'or où ils étaient bien portants et mangeaient à leur faim, comme le confirme le témoignage de ce vieux pasteur peul rencontré dans un village du Ferlo :

L'alimentation d'avant était meilleure. Les gens mangeaient à leur faim. Aujourd'hui, nos enfants ont faim. Les docteurs disent que le riz est le responsable de nos maladies. Il y a beaucoup de gens qu'on a interdit de manger du riz. Même moi, j'en fais partie. Je suis en train de mourir de faim. Et je ne vois pas de mil. 
Comme on le voit dans ce discours, le riz est accusé par la plupart de nos interviewés d'être responsable de nombreux maux comme le diabète, les ballonnements, la constipation, l'amaigrissement, l'indigestion. Dans les années 1980, Henri Barral et al. (1983) avaient déjà montré que $29 \%$ de la population totale du Ferlo était anémiée. Selon leur étude, les hommes étaient moins touchés $(14,7 \%)$ et les enfants âgés de 0 à 5 ans étaient les plus touchés $(41 \%)$. Aujourd'hui, la prévalence du diabète dans notre zone de recherche est de 4,2\% (Duboz et al. 2017). Les auteurs de l'étude estiment que ce sont probablement les changements rapides dans le régime alimentaire qui ont progressivement conduit à une augmentation du diabète au cours des 30 dernières années dans cette région rurale du Sénégal. Ce riz qui était, à l'époque coloniale, une denrée rare et recherchée est devenu aujourd'hui un « aliment à controverse », déprécié par tous pour ses aspects « négatifs », mais tout de même consommé par chacun. C'est aussi le cas, typiquement, du sucre : depuis son apparition en Occident, il a été alternativement angélisé et diabolisé (Fischler 2001). Ainsi, que l'on considère la ville ou la brousse, un paradoxe apparaît en ce qui a trait à l'alimentation dite « moderne ».

\section{Une dépendance aux « goûts de la ville »}

\section{Usage des bouillons cubes et volonté d'émancipation}

Les bouillons en poudre utilisés pour augmenter la sapidité des mets sont devenus incontournables dans l'alimentation des Sénégalais depuis leur introduction progressive par les colons français (Lepidi 2015). Aujourd'hui, des multinationales sont bien implantées au Sénégal et leur présence est très visible dans les messages et sur les panneaux publicitaires. Au Ferlo, la propagation des bouillons cubes a été facilitée par l'ouverture des marchés hebdomadaires et l'accès aux centres urbains favorisé par les voitures tout-terrain transportant humains et marchandises. Avant la grande sécheresse de 1972-1973, les populations du Ferlo utilisaient des fleurs de Balanites aegyptiaca, les fruits verts de Leptdenia hastata pour relever le goût de leurs sauces. Mais depuis qu'elles ont connu les bouillons cubes, elles ont abandonné la cueillette des plantes sauvages pour assaisonner leurs plats. À partir des années 1970, la présence répétée des agents des projets de développement, la migration vers la ville et la valorisation à Dakar et Saint-Louis ${ }^{11} \mathrm{du}$ « manger occidental » vont conforter leur adoption jusqu'à aujourd'hui. Facile à conserver, au coût peu élevé, le bouillon cube permet une fantaisie individuelle et pallie le manque de goût de viande ou de poisson, denrées financièrement inaccessibles. Nos enquêtes sur l'usage des plantes dans ces familles traduisent bien ce besoin ressenti par les femmes de mettre à distance « l'environnement naturel », tant pour ne pas apparaître comme « arriérées » que parce que l'argent régit désormais les échanges et, enfin, parce

11. Saint-Louis a été la capitale du Sénégal jusqu'en 1957. De 1895 à 1902, Saint-Louis a également été la capitale de l'Afrique-Occidentale française. 
que les bouillons cubes ont « le goût de la ville », de l'émancipation individuelle (Ka et al. 2016 ; Crenn et Ka 2019).

\section{Le bouillon de tous les dangers}

Aujourd'hui, les populations du Ferlo utilisent largement les bouillons cubes, même si elles les jugent nocifs et responsables de l'existence de maladies comme l'hypertension artérielle, l'ulcère de l'estomac, les cancers, les troubles érectiles, les crises cardiaques :

On souffre de fièvre, de courbatures, de problèmes de vision, de douleurs articulaires. Tout le monde souffre d'hypertension artérielle. Avant, on ne connaissait pas ces maladies. C'est dû aux bouillons en poudre. Le [bouillon cube] Jumbo n'est pas bon pour la santé.

\section{Homme, pasteur, 63 ans}

En effet, une étude sur la pression artérielle menée par Maïmouna Touré et al. (2012) montre une prévalence importante d'hypertension artérielle dans la population du Ferlo, soit chez 38,5\% de la population totale. Toujours selon Touré et al., la prévalence des maladies cardiovasculaires, qui incluent l'hypertension artérielle, dans la population globale du Ferlo atteint 13,4 \%. Malgré les risques supposés que l'usage des bouillons cubes implique, les populations continuent toujours à consommer avec délectation ces exhausteurs de goût. Les plus âgés justifient ce comportement paradoxal par la paresse des ménagères, qui ne veulent plus aller chercher des plantes sauvages dans la brousse pour épicer leur cuisine. Mais cela s'explique également par l'accessibilité géographique et financière des bouillons cubes : le marché est gorgé de ces derniers ; le cube de 10 grammes coûte 25 francs $\mathrm{CFA}^{12}$.

$\mathrm{Au}$ Ferlo, les bouillons cubes seraient utilisés pour stériliser les bœufs, les béliers, les boucs et les ânes. Selon certains de nos enquêtés, les pasteurs font avaler à leurs animaux mâles des solutions à base de ces bouillons cubes pour les rendre impuissants, comme en témoignent les propos de ce pasteur peul :

Les bouillons cubes causent des troubles érectiles. La preuve : aujourd'hui, pour castrer un bouc, on dissout cinq [cubes de bouillon] Jumbo dans un quart de litre d'eau et on le fait boire à l'animal.

Homme, pasteur, 48 ans

Cette stérilisation « chimique » à partir de bouillon en poudre alimente la rumeur sur sa nocivité. Cela laisse toutefois perplexes certains de nos interlocuteurs. Les propos de ce pasteur peul en sont une illustration :

12. Un euro équivaut à 656 francs de la Communauté financière africaine (francs CFA) ; 25 francs CFA équivalent à 0,06 dollars canadiens au moment d'écrire ces lignes. 
Certains accusent les bouillons. Mais je n'en suis pas sûr parce qu'il devrait y avoir des experts qui veillent sur la santé des populations. Ils ne doivent pas laisser les usines fabriquer des aliments nocifs. Mais j'ai constaté l'existence de maladies que je ne connaissais pas avant, en l'occurrence les cancers, les crises cardiaques.

Homme, pasteur, 53 ans

Selon Sophie Barro (2013), les bouillons en poudre contiennent une grande quantité de sel et du glutamate de potassium, deux substances favorisant les maladies cardiovasculaires. Les résultats d'un travail plus récent sur cette même population du Ferlo nous indiquent que les hommes et les femmes de 50 ans hypertendus représentent respectivement $65 \%$ et $60 \%$ de leur classe d'âge. Les valeurs de la pression artérielle mesurées lors des consultations sont parfois très élevées, soit $265 \mathrm{mmHg}$ de pression systolique et 140 de pression diastolique, d'où un risque important d'accident vasculaire à court terme (Cocaul-André 2015).

\section{Conclusion}

Les aliments, les manières de cuisiner, les goûts suivent l'histoire politique, économique et écologique des peuples. Le Ferlo, considéré par bon nombre de Sénégalais comme une enclave, loin de la culture urbaine, n'a jamais fonctionné en vase clos, hors du temps. Contrairement aux idées reçues qui en font fréquemment des individus appartenant à un monde révolu, arriéré, fixe, les Peuls du Ferlo suivent des itinéraires alimentaires pluriels et dynamiques. En effet, j'ai pu constater que les répertoires et habitudes culinaires varient selon la position sociale occupée sur ce territoire et dans la société locale. Les comportements alimentaires sont aussi marqués par le genre, l'âge et le lieu où l'on réside (vivre dans le bourg n'induit pas les mêmes pratiques que vivre dans un campement qui en est souvent éloigné de plus d'une dizaine de kilomètres). Les répertoires du comestible varient également sur ce territoire en fonction des croyances religieuses ou magico-religieuses (guérisseurs) et des valeurs éthiques (écologisme des développeurs) et des expériences culinaires capitalisées depuis l'enfance (éducation culinaire, transhumances, migrations en ville ou à l'étranger).

Ce que j'ai également pu constater, c'est que les mangeurs de cette partie du Ferlo réadaptent leur alimentation en fonction de l'influence culinaire des nouveaux acteurs sociaux qu'ils côtoient, en dépassant des normes acceptées jusque-là ou en découvrant de nouvelles manières de manger. Bref, dans cette zone sahélienne, les mangeurs rencontrés suivent et créent des parcours alimentaires multiples, choisissent des marqueurs identitaires qui les différencient les uns des autres tout en créant et en renforçant des appartenances et des liens sociaux dans un contexte sociohistorique par la forte présence de projets de développement sur leur territoire. 
L'implantation de marchés hebdomadaires, de boutiques, de gargotes ou encore de boulangeries artisanales a modifié en profondeur le régime alimentaire des Peuls. Le riz se substitue progressivement au mil, dont la disponibilité est de plus en plus incertaine. Mais il devient également plus prestigieux de consommer des produits venus de l'extérieur. Les divers légumes cultivés dans les jardins polyvalents de la Grande muraille verte sont aussi présents. Les produits venus de la ville et consommés par les agents des projets de développement ou les fonctionnaires de l'État sénégalais responsables des politiques publiques sont valorisés. Ces salariés installés temporairement dans le Ferlo apportent de nouveaux aliments, de nouveaux goûts, de nouvelles idées.

Les habitants autochtones s'approprient ces nouveautés pour diverses raisons : le riz serait plus facile à préparer que le mil ou le goût des bouillons cubes, meilleur que celui des plantes servant de condiments du Ferlo. L'accessibilité, le goût de la " modernité » sont souvent mis en avant par les femmes pour expliquer l'adoption des produits alimentaires usinés. En même temps émerge la critique de ces aliments relevant d'une alimentation urbaine perçue comme risquée et provoquant des maladies. En outre, cette critique recèle en filigrane la nostalgie d'une époque d'abondance idéalisée (le Ferlo d'avant la grande sécheresse de 1972-1973). Elle interroge également le rôle des femmes dans l'émergence de ces nouvelles pratiques alimentaires. C'est pourquoi cette recherche sur le terrain du Ferlo, prenant le contre-pied de l'idée d'un modèle culinaire stable et hermétique aux transformations du monde, permet au contraire d'en dévoiler certaines tensions et certaines formes de dépendance qui sont liées en particulier à l'importation de pratiques alimentaires mondialisées.

\section{Remerciements}

Ce travail a bénéficié d'une aide de l'État français gérée par l'Agence nationale de la recherche au titre du Labex DRIIHM, programme Investissements d'avenir portant la référence ANR-11-LABX-0010, et du soutien du projet Milkyway et de la Fondation Véolia Environnement. Je tiens également à remercier Chantal Crenn (Université Bordeaux Montaigne), Gilles Boëtsch et Enguerran Macia (UMI 3189 ESS-CNRS) pour leur appui scientifique.

\section{Références}

Appadurai A., 1996, Modernity at Large: Cultural Dimensions of Globalization. Minneapolis, University of Minnesota Press.

BA B., 2008, Agriculture et sécurité alimentaire au Sénégal. Paris, L’Harmattan. 
Barral H., E. Bénéfice, G. Bouget, J.-P. de Wispelaere, I. Diaite, O. T. Diaw, K. Dieye, M. P. Doutre, J.-F. Meyer, J. Noël, G. Parent, J. Piot, D. Planchenault, C. Santoir, C. Valentin, J. Valenza et G. Vassiliades, 1983, « Systèmes de production d'élevage au Sénégal dans la région du Ferlo : synthèse de fin d'études d'une équipe de recherches pluridisciplinaire ». Dakar, Ministère de la Recherche et de l'Industrie et GERDATORSTOM.

Barro S., 2013, "Les bouillons contiennent du potassium qui bloque le cœur », L'Observateur, 2944 : 5.

Benoit M., 1988, « La lisière du Kooyaa. Espace pastoral et paysage dans le Nord du Sénégal (Ferlo) », L'Espace géographique, 2 : 95-108.

Bessis S., 1981, L'arme alimentaire. Paris, Maspero.

Bonfiglioli A., 1988, Dudal. Histoire de famille et histoire de troupeau chez un groupe de Wodaabe du Niger. Paris, Éditions de la Maison des sciences de l'homme.

BonneCASE V., 2009, « Avoir faim en AOF. Investigations et représentations coloniales (19201960) », Revue d'histoire des sciences humaines, 21 : 151-174.

Cocaul-André M., 2015, Hypertension artérielle et composition corporelle chez les Peuls de la région de Téssékéré. Mémoire de master II, anthropologie biologique, Université Cheikh Anta Diop de Dakar.

Combessie J.-C., 2007, La méthode en sociologie. Paris, La Découverte.

Corbeau J.-P., 1994, « Goûts des sages, sages dégoûts, métissage des goûts » : 164-182, in Le métis culturel. Arles, Actes Sud et Paris, Maison des cultures du monde.

Corbeau J.-P. et J.-P. Poulain, 2002, Penser l'alimentation. Entre imaginaire et rationalité. Toulouse, Privat.

Crenn C. et J.-P. Hassoun, 2014, « Dakar. Les quatre âges du fast-food », Ethnologie française, 44 : 59-72.

Crenn C. et A. KA, 2012, " "En quête" d'alimentation dans la zone Téssékéré/Widou au Sénégal ", Les Cahiers de l'Observateur hommes-milieux international Téssékéré, 2 : $37-48$.

—, 2019, «Foodscape entre migrations et circulations » : 201-212, in G. Boëtsch, P. Duboz, A. Guisse et P. Sarr (dir.), La grande muraille verte. Une réponse africaine au changement climatique. Paris, CNRS Éditions.

Crenn C., A. Ka et J. Leport, 2015, « Entre brousse, ville et cadre de la globalisation : les paysages alimentaires du Sénégal »: 171-202, in G. Boëtsch, E. Macia, L. Gueye et A. Guissé (dir.), Santé et société en Afrique de l'Ouest. Paris, CNRS Éditions.

De Garine I., 1962, « Usages alimentaires dans la région de Khombole (Sénégal) », Cahiers d'études africaines, $10: 218-265$.

Demante M.-J., 2006, Appui à la capitalisation des expériences du Projet d'autopromotion pastorale dans le Ferlo. Saint-Louis, PAPF.

Dıop B., 2013, L'influence de la publicité alimentaire sur la consommation au Sénégal : le cas des bouillons en poudre. Mémoire de master II, Université Toulouse II-Le Mirail. 
Diop C. A., 1954, Nations nègres et culture. Paris, Présence africaine.

Duboz P., G. Boёtsch, L. Gueye et E. Macia, 2016, « Hypertension in the Ferlo (Northern Senegal): Prevalence, Awareness, Treatment and Control ", consulté sur Internet (http://www.panafrican-med-journal.com/content/article/25/177/pdf/177.pdf), le 13 octobre 2017.

Duboz P., G. Bö̈tsch, L. Gueye et E. Macia, 2017, « Type 2 Diabetes in a Senegalese Rural Area », World J Diabetes, $8:$ 351-357.

Evans-Pritchard E. E., 1940, The Nuer: A Description of the Modes of Livelihood and Political Institutions of a Nilotic People. Oxford, Clarendon Press.

FisChleR C., 1979, « Gastro-nomie et gastro-anomie », Communications, 31 : 189-210.

—, 2001, L'Homnivore. Paris, Odile Jacob.

Gado B. A., 1993, Une histoire des famines au Sahel. Étude des grandes crises alimentaires (XIX $X^{e}-X X^{e}$ siècles). Paris, L'Harmattan.

KA A., 2016, Manger à Widou Thiengoly (Nord-Sénégal) : de l'abondance remémorée à la dépendance au marché. Thèse de doctorat, École doctorale "Sciences de l'Homme et de la Société », Université Cheikh Anta Diop.

Ka A., L. Ndiaye et C. Crenn, 2016, « Circulation, dénigrement des savoir-faire autour des plantes médicinales dans le Ferlo », Ethnopharmacologia, 56 : 53-57.

KenYatta J., 1938, Facint Mount Kenya. Londres, Seeker and Warburg.

Lam A. B., 1993, De l'origine égyptienne des Peuls. Paris, Présence africaine.

LePIDi P., 2015, « En Afrique, le cube Maggi à toutes les sauces », Le Monde, 6 mars 2015, consulté sur Internet (http://www.lemonde.fr/afrique/article/2015/03/06/en-afrique-ducube-maggi-a-toutes-les-sauces_4588992_3212.html), le 18 février 2018.

Macia E., P. Duboz et L. Gueye, 2010, « Les dimensions de la qualité de vie subjective à Dakar », Sciences sociales et santé, $28: 75-84$.

Macia E., L. Gueye et P. Duboz, 2016, « Hypertension and Obesity in Dakar, Senegal », consulté sur Internet (https://journals.plos.org/plosone/article?id=10.1371/journal. pone.0161544), le 3 août 2017.

Manoli C. et V. Ancey, 2013, «Effets ambigus de la mobilité des jeunes pasteurs : entre la sécurisation des conditions de vie pastorales et l'émancipation individuelle au Ferlo, Sénégal » : 185-201, in G. Azoulay, V. Ancey, D. Dormoy, C. Crenn, A. Mangu et A. Thomashausen (dir.), Mobilités et migrations : figures et enjeux contemporains. Pluralité des regards et des disciplines. Paris, L'Harmattan.

Mintz S., 1991, Sucre blanc, misère noire. Le goût et le pouvoir. Paris, Nathan.

Ninot O., 2003, Vie de relations, organisation de l'espace et développement en Afrique de l'Ouest : la région de Tambacounda au Sénégal. Thèse de doctorat, UFR Lettres et Sciences humaines, Université de Rouen. 
O'DÉYÉ M., 1985, « À propos de l'évolution des styles alimentaires à Dakar » : 179-195, in N. Bricas, G. Courade, J. Coussy, P. Hugon et J. Muchnik (dir.), Nourrir les villes en Afrique sub-saharienne. Paris, L'Harmattan.

Pales L. et M. Tassin de Saint Peureuse, 1955, L'alimentation en A.O.F. Milieux Enquêtes - Techniques - Rations. Dakar, ORANA.

Poulain J.-P. et L. Tibère, 2000, « Mondialisation, métissage et créolisation alimentaire. De l'intérêt du "laboratoire" réunionnais », Bastidiana, 31-32 : 225-242.

Richards A., 1932, Hunger and Work in a Savage Tribe. A Functional Study of Nutrition Among the Southern Bantu. Londres, Routledge.

SArrouy C., 2010, Insécurité alimentaire au Sénégal : l'agro-écologie comme réponse à la sous-alimentation et à la dégradation de l'environnement dans un pays en développement. Mémoire de master, Université libre de Bruxelles.

Tercier S. N. et B. Sottas, 2000, La sécurité alimentaire en questions. Paris, Karthala.

Touré M., A. Brus, G. Bö̈Tsch et L. GueYe, 2012, «Étude sociodémographique, anthropologique et épidémiologique de la population du Ferlo », Les Cahiers de l'Observateur homme-milieux international Téssékéré, 2 : 9-22.

Yount C., 2010, Tout a changé, sauf le repas : les habitudes alimentaires dakaroises face aux enjeux socio-économiques. Mémoire de master, Centre Edgar Morin, École des hautes études en sciences sociales.

\section{RÉSUMÉ - ABSTRACT-RESUMEN}

Manger au Ferlo (Sénégal). Les charmes risqués des " goûts de la ville »

Les villages où l'auteur a recueilli les données alimentaires dont il est question dans cet article se situent dans la zone sylvopastorale et semi-aride du Ferlo, au nord du Sénégal. Les projets de développement et de lutte contre la désertification s'y suivent depuis les années 1970. La population est majoritairement peule, mais certaines familles sont également wolofs ou maures. À partir d'une enquête ethnographique approfondie, l'auteur met en lumière les tensions que suscite l'introduction de produits alimentaires industriels venus de Dakar ou de l'étranger dans ce Ferlo autrefois habitué à l'autosubsistance (mil, lait, plantes alimentaires sauvages). Il insiste sur la valeur symbolique de ces produits, mais aussi sur leurs ambivalences. Ainsi, le riz et le bouillon cube venus de l'extérieur sont tous deux porteurs de risques sanitaires compte tenu de leur apport excessif en sucre et en sel. L'auteur s'interroge en creux sur les nouvelles formes de dépendance alimentaire que cette situation induit.

Mots clés : Ka, Sénégal, Ferlo, Peul, alimentation, santé, riz, bouillon cube, anthropologie

Eating in Ferlo (Senegal). The Risky Charms of the "Tastes of the City"

The villages where the author collected the facts about food discussed here are located in the sylvo-pastoral and semi-arid area in northern Senegal (Ferlo). From 1970, several projects for development and against desertification have been conducted. The Fulani make 
up most of the population but some families are also Wolof or Moorish. Based on an intensive ethnographic survey, the author clarifies the tensions raised by the introduction of industrial foodstuffs from Dakar or elsewhere into this Ferlo once used to self-sufficiency (millet, milk, Wild food plants). The author insists on the symbolic value that surrounds these products but also in their ambivalences. Thus, the rice and the bouillon cube coming from the outside are both carriers of health risks given their excessive intake of sugar and salt. He questions the new forms of food dependence that this situation induces.

Keywords: Ka, Senegal, Ferlo, Fulani, food, health, rice, bouillon cube, anthropology

\title{
Comer en el Ferlo (Senegal). Las delicias arriesgadas de los «sabores de la ciudad»
}

Los pueblos en donde el autor recogió los datos alimentarios que aquí se tratan se sitúan en la zona silvopastoral y semi-árida del norte de Senegal: el Ferlo. Ahí, los proyectos de desarrollo y de lucha contra la desertificación se han llevado a cabo desde los años 1970. Los Peuls constituyen la mayoría de la población pero hay algunas familias Wólof o Moros. A partir de una investigación etnográfica profunda, el autor muestra las tensiones que provoca la introducción de productos alimenticios industriales provenientes de Dakar o del extranjero en ese Ferlo antes habituado a la autosuficiencia alimentaria (mijo, leche, plantas comestibles salvajes). Se subraya el valor simbólico que envuelve a dichos productos, pero también a sus ambivalencias. Así pues, el arroz y el cubo de consomé provenientes del exterior son ambos portadores de riesgos sanitarios, debido a su excesivo aporte de azúcar y sal. El autor cuestiona las nuevas formas de dependencia alimentaria que esta situación provoca.

Palabras clave: Ka, Seneral, Ferlo, Peul, alimentación, salud, arroz, cubo de consomé, antropología

\author{
Abdou Ka \\ Département de sociologie \\ Université Assane SECK de Ziguinchor \\ UFR des Sciences économiques et sociales \\ BP 523 \\ Ziguinchor \\ Sénégal \\ UMI 3189 Environnement, Santé, Sociétés (CNRS) \\ asska18@hotmail.com
}

